\title{
RECONOCIENDO LOS DESASTRES SOCIO-NATURALES OCURRIDOS EN MÉXICO
}

\author{
Luis Miguel Espinosa Rodríguez ${ }^{1^{*}}$
}

\section{RESUMEN}

Con base en documentos históricos, el presente trabajo recopila eventos calamitosos ocurridos en México reportados a partir de la época prehispánica y clasificados de acuerdo a su origen. A través de la realización de una crónica referencial, se establece una tipología con las siguientes amenazas: sísmicos, tsunamis, volcánicos, e hidrometeorológicos, como huracanes, tormentas tropicales, inundaciones, sequías, precipitación extraordinaria y tornados. La metodología general se circunscribe a la Ecuación General de Riesgo (EGR), y se discute la presencia histórica de los desastres versus el crecimiento general de la población y la necesidad de la generación de fundamentación de políticas públicas orientadas a salvaguardar el patrimonio humano y material. Los resultados muestran la necesidad de reconocer el conjunto de desastres de origen natural que se han presentado en el territorio mexicano desde una perspectiva histórica, apuntando a que la población y el gobierno puedan accionar de manera conjunta en un esquema real de gestión integral de los riesgos de desastre en el futuro. En especial en un escenario de aumento de la población, la cual ocupará territorios susceptibles al desarrollo de geosistemas perturbadores, condición que aumentará la exposición y la vulnerabilidad de más de 65 millones de personas en 2040.

\section{PALABRAS CLAVES}

Desastres, Amenazas, Población, Políticas públicas, México

\section{RECOGNIZING SOCIO-NATURAL DISASTERS OCCURRED IN MEXICO}

\section{ABSTRACT}

Based on historical documents, this work compiles disaster events occurred in Mexico from pre-Hispanic times and classified according to their origin. Through the realization of a historicreferential technique, a typology is established with the following hazards: seismic, tsunamis, volcanic, and hydrometeorological such as tropical storms, floods, droughts, and tornadoes. The general methodology relates to the General Risk Equation (EGR), which addresses the historical presence of disasters versus the general growth of the population and the need to generate better public policies. The results show the need for recognizing the wider set of disasters of natural origin that have occurred in the Mexican territory and from a historical perspective, pointing out that the population and the government should act together in a real scheme of comprehensive risk management disaster in the future. In a scenario of population growth and territories susceptible to the development of disturbing geosystems, this may increase the exposure and vulnerability of more than 65 million people by 2040 .

\section{KEYWORDS}

Disasters, Hazards, Population, Public policies, Mexico
1. Facultad de Geografia, Universidad Autónoma del Estado de México, Toluca, México.

*Autor de correspondencia: geo_luismiguel@hotmail.com

Identificador:

http://revistareder.com/

handle-0719-8477-2021-069

\section{RECIBIDO}

23 de mayo de 2020

ACEPTADO

14 de septiembre de 2020

PUBLICADO

1 de enero de 2021

Formato cita

Recomendada (APA): Espinosa Rodríguez, L.M. (2021). Reconociendo los desastres socio-naturales ocurridos en México. Revista de Estudios

Latinoamericanos sobre Reducción del Riesgo de Desastres REDER, 5(1), 96109. http://revistareder.com/ handle-0719-8477-2021-069

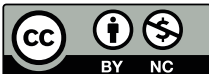

Todos los artículos publicados en REDER siguen una política de Acceso Abierto y se respaldan en una Licencia CreativeCommons Atribución-NoComercial 4.0 Internacional.

Revista de Estudios Latinoamericanos sobre Reducción del Riesgo de Desastres (REDER) 


\section{INTRODUCCIÓN}

Desde una perspectiva particular, la vulnerabilidad se define como una serie de variables qué influyen de manera directa en el impacto de un subsistema perturbador específico, y de las cuales se destacan aspectos relacionados con el territorio cómo puede ser la geomorfología asociada a pendientes abruptas, materiales poco consolidados, morfología de cauces, así como la localización física de la población, aspectos de índole cultural, ideológico y educativo entre otros. En su conjunto, éstos factores de vulnerabilidad pueden provocar -con alta probabilidad-, que un evento extraordinario represente impacto alto en los grupos de población y el medio natural.

De acuerdo con anterior el riesgo se circunscribe a la integración compleja de diferentes variables que componen en primer término a un geosistema perturbador y los de enlace (o encadenados) que se asocian a éste, los cuales pueden poseer un origen natural (tectónico, volcánico, gravitacional, atmosférico); o también origen social referido a manifestaciones organizativas de diversa índole.

Asimismo, el riesgo pose un componente fundamental qué es el territorio en el cual y sobre el cual se desarrollan los procesos antes descritos, y que de manera particular sobre este queda enclavado el factor más importante: el factor humano; el cual posee diversas cualidades y características que lo pueden hacer más o menos susceptible y/o vulnerable ante presiones internas y externas que modifican e impactan la morfología y funcionamiento.

En este tenor, este trabajo pretende reconocer el conjunto de desastres de origen natural ocurridos en México desde un perspectiva histórica. El trabajo se relacionan con variables que en conjunto pueden afectar el nivel de vulnerabilidad de la sociedad mexicana, en el entendido de que si bien se reconocen algunos eventos catastróficos, ello sólo se hace en el ámbito local y se generaliza acerca de ellos: a esta condición el factor "tiempo" determina condiciones de olvido colectivo que pueden ser peligrosas ante la ocurrencia de un evento; por ejemplo las nuevas generaciones difícilmente tienen consciencia del desastre ocurrido en 1985 y los sucesos representan un dato o hecho anecdótico.

De acuerdo con lo anterior se considera que la relación entre las variables que constituyen al riesgo es compleja; y que ésta aumenta cuando el número de pobladores expuestos es alto; así, en un esfuerzo por sintetizar el contexto nacional se parte de la premisa: "A mayor aumento de la población, y mayor densidad de ésta; mayor es la exposición a geosistemas perturbadores y la probabilidad de riesgo aumenta" (Figura 1).

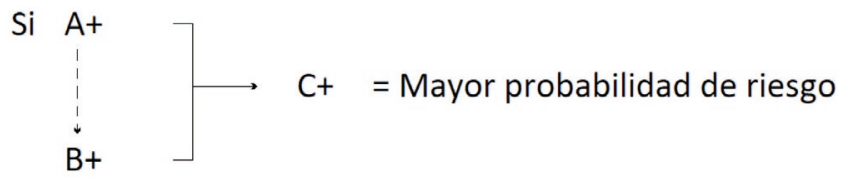

Figura 1. Relación entre las variables de número de población (A), densidad (B) y exposición-vulnerabilidad (C) Fuente: Autor, 2021.

Finalmente, el trabajo elabora un catastro de desastres que se relaciona con la tesis que postula que el desconocimiento de los sucesos de origen natural qué provocan peligros, aumenta la vulnerabilidad y a razón del incremento poblacional.

La configuración del territorio nacional, la localización geográfica, así como la evolución geológica, geomorfológica y climática; el desarrollo de eventos históricos (rebeliones, explosiones, etcétera), así como acontecimientos de orden natural como los meteorológicos y morfodinámicos entre otros, y la situación general de pobreza, marginación y educación entre otros factores, han escrito y dejado en el territorio huellas de desastres que además de propiciar pérdidas tangibles e intangibles; y aseguran condiciones propicias para la conservación y aumento de la vulnerabilidad social en la medida de que se incrementa el número de la población en nuestro país.

Los primeros registros concernientes a desastres de orden natural, social y sanitarios datan de la época prehispánica en donde se encuentran algunas referencias en torno a la Albarrada de Netzahualcóyotl o "De los indios" que fue construida para proteger y regular de las inundaciones a la Gran Tenochtitlan; así como anaglifos que representaban la ocurrencia de sismos durante el día o la noche y que relacionaban con dioses como Tezcatlipoca (para los Aztecas) o los Pahua tunes que eran los sostenedores del plano de la Tierra para los mayas. 
Durante el periodo de la Conquista y la Colonia se escribieron pliegos como el Códice Telleriano Remensis, documento que da cuenta de un grupo de sismos ocurridos antes del año de 1455, así como los textos: Aubin, Matritense del Real Palacio, Chimalpopoca; y los Anales de: Tlatelolco, Cuautitlán, Tepaneca, de Quecholac, y el de Puebla y Tlaxcala publicados en diferentes años entre otros. A estos, legajos se les suman los diferentes "Diarios de sucesos notables y curiosos", así como algunos expedientes realizados en diversos obispados del país que registran datos de mortandad.

De acuerdo con lo anterior, el objetivo de este artículo se centra en primer término, en la realización de una crónica referencial de los procesos que han generado socio-naturales en el territorio mexicano, considerando referencias históricas que parten del periodo prehispánico hasta el tiempo presente. Esto es lo que entenderemos como un catastro.

Clasificar al grupo de eventos catastróficos que más han afectado a nuestro país, tiene como propósito la identificación de las variables que favorecen el incremento de la vulnerabilidad más comunes que se presentan en el territorio nacional con la finalidad de comprender los puntos más débiles de los sistemas que provocan riesgos y exponer las tendencias de éstos para ayudar a la conformación de políticas públicas.

\section{METODOLOGÍA}

La metodología planteada se basa en una revisión bibliográfica exhaustiva que incluye algunas notas periodísticas. Con el propósito de acceder a una búsqueda, selección, recopilación y análisis de fuentes bibliográficas de primer orden, se hizo el acopio y documentación de la información de acuerdo con la temas seleccionados para la investigación, seleccionándose los tópicos de riesgos naturales: sismicidad, vulcanismo y deslizamientos; socio-organizativos que incluyen aspectos relacionados con sanidad, represión de movimientos obreros y estudiantiles, terrorismo y grupos de choque, guerras internas, ataques a la población, incidentes y percances.

Se llevó a cabo un estudio de las características generales de los archivos y documentos (bibliográficos y hemerográficos) para con ello realizar el balance entre la calidad de las fuentes, los vacíos de información, y determinar con ello el alcance y los límites de la investigación.

Una vez realizado el análisis de los formatos y fichas informativas de los eventos históricos de los desastres ocurridos en México, se sistematizó la información clasificándose en temáticas y categorizando los subtipos de ésta y; con ello, se identificaron los textos que cumplieran especificaciones de originalidad en el mayor número de los casos y; en caso de existir lagunas informativas. Asimismo, en el caso de fuentes documentales que se encuentran en la red de internet, se buscaron y verificaron los URL de los textos que se encuentran en páginas como Google Académico, aún en los casos en donde existen fuentes documentales impresas.

La metodología general que se ha seguido se circunscribe a la Ecuación General de Riesgo (EGR propuesta para México por Espinosa y Hernández (2015), de la cual para este trabajo de investigación se retoma sólo la función del Geosistema perturbador y las variables que lo conforman (como los geosistemas de enlace o encadenados, los factores relieve y hemerobia, la capacidad científica, técnica y social de respuesta, la resiliencia y el factor multivariable: análisis potenciado representado por el exponencial (" $n+3 ")$; entendido todo ello como parte de un subsistema que se integra a la función humana, la función del territorio, la función de la gestión y la función sistémica.

En la EGR se plantea que el riesgo posee cinco variables sustantivas: la función del geosistema perturbador (fGP), el componente humano $(\mathrm{fH})$, el factor territorio ( $\mathrm{fT}$ ), el funcionamiento sistémico (fS), y el factor de gestión ( $\mathrm{fG}$ ), con las cuales se deriva el análisis de la vulnerabilidad y la exposición (Tabla 1). 


\begin{tabular}{|c|c|c|}
\hline Factor & Variable & Ejemplos de criterios de evaluación \\
\hline \multirow{4}{*}{ GP } & Geosistema perturbador (GP) & $\begin{array}{l}\text { Energía medida por el Servicio Sismológico Nacional (SSN) y la US Geological } \\
\text { Survey (USGS) }\end{array}$ \\
\hline & Factor relieve (FR) & Estratigrafía y aceleración sísmica \\
\hline & Factor de hemerobia $(\mathrm{H})$ & Metodología de Quadri (2000; en Ortiz y Mateo, 2010) \\
\hline & Geosistema(s) encadenado(s) (gE) & $\begin{array}{l}\text { Valoración paramétrica de acuerdo Tricart (1990), Palacio, 1995; Espinosa y } \\
\text { Hernández (2014) }\end{array}$ \\
\hline \multirow{12}{*}{$\mathrm{FH}$} & Percepción científica (Pc) & Valoración a través del número de publicaciones cientificas \\
\hline & Percepción social y psicológica (Ps) & Encuesta general basada en la Teoría de la Gestalt \\
\hline & Factor educación (Fe) & $\begin{array}{l}\text { Valoración a través de programas y contenidos educativos formales e informales } \\
\text { (escolarizados o no) }\end{array}$ \\
\hline & Factores socioeconómicos y políticos (Fs) & A través de información de INEGI y El Financiero \\
\hline & Medidas estructurales (MedE) & Estudio de infraestructura en campo \\
\hline & Estructura familiar (Ef) & Valoración a través de información de INEGI \\
\hline & Percepción del orden de gobierno $(\mathrm{Pg})$ & A través de la planeación en programas y acciones específicas \\
\hline & Voluntad política y factor de corrupción (Vp) & Ejecución y calidad de programas y acciones específicas \\
\hline & Aceptación social del riesgo (As) & Levantamiento en campo (entrevistas y encuestas) \\
\hline & Adaptación social del riesgo (Ad) & Levantamiento en campo (entrevistas y encuestas) \\
\hline & Comunicación social del riesgo (Cs) & Revisión de programas de difusión (oficial y comercial) \\
\hline & Medidas no estructurales (MnE) & Revisión de programas de prevención \\
\hline \multirow{8}{*}{ FT } & Seguridad y valoración financiera (Sf) & Análisis de paquetes y programas de seguridad \\
\hline & Exposición y susceptibilidad del territorio (Ex) & Análisis geográfico regional y local \\
\hline & Valor del territorio (Vt) & Levantamiento en campo (entrevistas y encuestas) \\
\hline & Valor de construcción social (Vcs) & Levantamiento en campo (entrevistas y encuestas) \\
\hline & Espacios escenciales (EE) & Aplicación de metodología modificada de Pigeon (2000) \\
\hline & Diagnosis $(\mathrm{Dg})$ & Aplicación metodológica de Ribas (en De Bolós, 1991) \\
\hline & Prognosis (Pgs) & Aplicación metodológica de Tura y Ribas (en De Bolós, 1991) \\
\hline & Sintéresis (St) & Aplicación metodológica de Tura y Ribas (en De Bolós, 1991) \\
\hline \multirow{2}{*}{ FS } & Proceso de retroalimentación (feedback) (Fd) & Análisis con base en Teoría General de Sistema y metodología de Pigeon (2000) \\
\hline & Funciones sistémicas & Análisis de variables de la Teoría General de Sistema \\
\hline \multirow{4}{*}{ FG } & Gestión de riesgo (GR) & $\begin{array}{l}\text { Metodología del Centro Naciomal para la Prevención de Desastres, CENAPRED } \\
(2006)\end{array}$ \\
\hline & Gestión integral de riesgo local de desastre (GI) & Metodología del CENAPRED (2006) \\
\hline & $\begin{array}{l}\text { Capacidad científica, tecnológica, social, } \\
\text { gubernamental de respuesta (Cr) }\end{array}$ & $\begin{array}{l}\text { Valoración a través del análisis de geosistema regulador (aplicaciones } \\
\text { específicas) y de programas de acción social }\end{array}$ \\
\hline & Resiliencia (Res) & Análisis con base en Teoría General de Sistema, entendida como autopoiesis \\
\hline
\end{tabular}

Tabla 1. Variables que componen a la Ecuación General de Riesgo y ejemplos de parametrización Fuente: Autor, 2021

\section{DESARROLLO}

\section{Eventos sísmicos}

De acuerdo con lo planteado, se presenta un registro de los eventos catastróficos que se han presentado en el país en el orden tipológico convencional; así y en primer término, aparecen los riesgos de origen geológico que refieren a la sismicidad.

Para este caso resulta fundamental comprender que México se encuentra emplazado sobre la Placa Americana, la cual se desplaza en dirección Norte y que, a su vez, es impactada por placas tectónicas que le comprimen y distienden con diferentes niveles de energía e intensidad temporal. La mayor parte de estos son de borde de placa e intraplaca.

En este marco se destaca la mecánica de la Placa de Cocos que subduce, gira en forma de arco y pivotea en la costa del Pacífico; la Placa Rivera localizada al Oeste del Estado de Jalisco y Colima que subduce e impacta al Bloque Jalisco y a la Junta triple; la Placa Pacífica que se 
desplaza en dirección NNW y que es el resultado del escindir de la antigua placa Farallón; y, la Placa del Caribe que desarrolla desplazamientos generalizados en dirección Oeste-Noreste que configuran la costa del Caribe.

En el ámbito regional se destaca la dinámica de sistemas de fallas normales, inversas y transcurrentes asociadas a bloques y/o estructuras desmembradas, horst y grabens que en conjunto se relacionan con morfoestructuras dominantes y sismicidad "local" como sucede en el Golfo de California; en el Sistema Volcánico Transversal, y el caso de los sistemas de fallas transcurrentes -"cola de caballo"- del Istmo de Tehuantepec entre otros ejemplos (Hernández et al., 1995).

La compleja dinámica de los sistemas territoriales ha propiciado la formación de regiones sismo-generadoras regionales y locales; las cuales han provocado movimientos telúricos de alto impacto a lo largo de la historia, de los cuales, se tienen pocos registros asociados a tsunamis (Tabla 2).

\begin{tabular}{|c|c|c|}
\hline Año & Evento (Nombre) & $\begin{array}{l}\text { Magnitud }\left(M_{w}\right) \\
\text { aproximada }\end{array}$ \\
\hline 1475 & Cuajimalpa y tsunami en el lago de Texcoco & 7.5 \\
\hline 1496 & Sismo del Año 4 pedernal & 7.7 \\
\hline 1542 & Ciudad de México & 7.0 \\
\hline 1589 & Coyoacán & SD \\
\hline 1611 & México y Xochimilco & SD \\
\hline 1619 & San Diego, Acapulco & SD \\
\hline 1653 & Atzcapotzalco & 6.9 \\
\hline 1665 & Tremor del Popocatépetl & 6.5 \\
\hline 1685 & Acapulco & 7.7 \\
\hline 1696 & Oaxaca & 7.5 \\
\hline 1697 & Acapulco & SD \\
\hline 1698 & México & SD \\
\hline 1701 & Oaxaca & 7.3 \\
\hline 1711 & Sismo de México, Puebla y Tlaxcala & 8.3 \\
\hline 1711 & Sismo de Colima & SD \\
\hline 1732 & De "la destrucción total del puerto de Acapulco" & 7.6 \\
\hline 1753 & ciudad de México & 7.3 \\
\hline 1754 & Acapulco & 7.6 \\
\hline 1768 & Atlixco, Ecatepec, Jamiltepec y México & 8.0 \\
\hline 1776 & Acapulco & SD \\
\hline 1787 & Costa de Guerrero y Oaxaca & 8.6 \\
\hline 1787 & Gran sismo de Guerrero y Oaxaca & 8.6 \\
\hline 1800 & Mixteca, Puebla y Veracruz & SD \\
\hline 1818 & Colima & 7.7 \\
\hline 1818 & Colima y Guadalajara & 7.3 \\
\hline 1820 & Acapulco y ciudad de México & 6.2 \\
\hline 1835 & Tlalnepantla & 7.0 \\
\hline 1837 & Brecha de Guerrero & 7.7 \\
\hline 1841 & Noreste de México (Monterrey, Tamaulipas y San Luis Potosí) & 7.0 \\
\hline 1845 & Brecha de Guerrero & SD \\
\hline 1854 & Sismo de Oaxaca & 8.0 \\
\hline 1858 & Texcoco & 7.7 \\
\hline
\end{tabular}




\begin{tabular}{|c|c|c|}
\hline Año & Evento (Nombre) & $\begin{array}{l}\text { Magnitud }\left(\mathrm{M}_{\mathrm{w}}\right) \\
\text { aproximada }\end{array}$ \\
\hline 1864 & Puebla y Veracruz & SD \\
\hline 1889 & Valle de Anáhuac & SD \\
\hline 1911 & Sismo "Maderista" & 7.8 \\
\hline 1912 & Enjambres de la Ciudad de Guadalajara (más de 3,000) & 6.4 \\
\hline 1912 & Acambay & 7.4 \\
\hline 1931 & Oaxaca & 7.8 \\
\hline 1932 & Costa de Jalisco y Colima "Cuyutlán*" & $8.4 ; 7.8$ y 6.9 \\
\hline 1932 & Tomatlán, Jalisco & 8.2 \\
\hline 1941 & Colima, Guerrero, Jalisco y Michoacán & SD \\
\hline 1957 & "Del Ángel" & 7.8 \\
\hline 1973 & Veracruz y Puebla & 8.3 \\
\hline 1979 & "De la lbero" & 7.6 \\
\hline 1985 & Costa de Guerrero y Michoacán; Ciudad de México & 8.4 y 7.4 \\
\hline 1995 & La Manzanilla Jalisco & 8 \\
\hline 1999 & Oaxaca & 7.0 \\
\hline 1999 & Puebla & 6.9 \\
\hline 2010 & Baja California & 7.2 \\
\hline 2014 & Chiapas & 6.9 \\
\hline 2017 & Cihuatlán Jalisco & 6.1 \\
\hline 2017 & Oaxaca y Chiapas & 8.2 \\
\hline 2017 & Sismo de Morelos (Ciudad de México, Puebla) & 7.2 \\
\hline
\end{tabular}

Tabla 2. Eventos sísmicos con magnitud superior a 6 grados de magnitud $\left(\mathrm{M}_{\mathrm{w}}\right)$

Fuente: Autor, 2021, con base en Pérez y García, 1989; Cenapred (2006), Valdivia y Castillo (2012); Díaz (2013), NGDC/WDS (2015); Levi y Toscana (2017)

\section{Eventos volcánicos}

México es conocido de manera popular como "El país de los volcanes", así como en el ámbito científico; ello debido a la conformación y dinámica de la provincia fisiográfica que ostenta el nombre de "Sistema volcánico Transversal", también conocida como "Eje volcánico", "Faja Volcánica Transmexicana" y "Eje Neovolcánico".

En este sistema destaca la presencia de estratovolcanes con actividad explosiva así como calderas de explosión y maares (axalapasgos); destacándose entre otros aparatos volcánicos el "Ceboruco", "Sanganguey" y "Santa María", localizados en el Estado de Nayarit; el volcán "Tequila" en Jalisco, la "Caldera de Amealco" y el "Cimatario" en el estado de Querétaro; los volcanes "Nevado de Toluca" y "Jocotitlán" en el estado de México; el volcán "Tancítaro" y "Jorullo" en Michoacán; el volcán "La Malinche" en Tlaxcala; así como el "Cofre de Perote" y "Pico de Orizaba", así como la "Caldera de los humeros" en Veracruz entre muchos otros.

A estos edificios se suman estructuras volcánicas producto de la interacción de sistemas de placas tectónicas y de sistemas de fallas regionales que provocaron zonas de debilidad cortical; y que por lo general representan áreas de alta complejidad estructural. Se destacan entre otros ejemplos el campo de "Cerro prieto" en Baja California; los volcanes costeros de Veracruz, "San Miguel" y "Santa Marta" en la región de los Tuxtlas, y el volcán "Tacaná" emplazado en el estado de Chiapas en el sureste de México.

Por lo que se refiere a las erupciones volcánicas más representativas en el periodo histórico, se encuentran las del estratovolcán Popocatépetl, el cual históricamente ha tenido registros de actividad durante los años 1289, 1354, 1947, 2000, 2003, 2005, 2011, 2012, 2013, 2017, 2018, 2019 y 2020; lo que implica un periodo de actividad que podría presentar una erupción de tipo pliniana o freatopliniana en el tiempo presente, motivo por el cual, el Centro Nacional de Prevención de Desastres (CENAPRED) mantiene vigilancia y monitoreo en tiempo real para aplicar programas 
alertamiento y evacuación; ello debido a que encuentra emplazado en los entidades estatales de Morelos, Puebla, México y Ciudad de México, condición que le relaciona con más dos millones de habitantes localizados en el piedemonte y zonas posibles de afectación.

Por su cuenta y también perteneciente al Sistema Volcánico Transversal, el Volcán de Fuego de Colima se emplaza en un complejo sistema estructural conocido como "la Junta triple" en donde se desarrolla el sistema de graben en Colima, el sistema de distensivo del lago de Chapala y, el complejo del Bloque Jalisco; sistemas regionales influenciados por la dinámica de la Placa Americana. La Placa Rivera y la Placa de Cocos. La mayor parte del edificio se encuentra emplazado en el estado de Jalisco y ha registrado actividad intensa en los años de 1576, 1585, 1606, 1622, 1890, 1903, 1913, 1991, 1999 y en las dos décadas del siglo XXI. Es monitoreado por el CENAPRED, así como por investigadores de loa Universidad de Coloma, la Universidad de Guadalajara y la Universidad Nacional Autónoma de México debido al alto valor de explosividad que posee (Robin et al., 1981 y 1991; Goff et al., 1988; Robin y Boudal, 1989; Garduño y Saucedo, 1998; Love et al., 1998; Siebe et al., 1995; Biondi et al., 2003; Alatorre, 2006).

El siglo pasado fue testigo de las erupciones del volcán Paricutín que se localiza en el Estado de Michoacán en 1943, y el Chichonal, localizado en el estado de Chiapas en 1982.

En el primer caso, la erupción se relaciona con la constitución del edificio volcánico en la localidad de San Juan Parangaricutiro y actividad de tipo vulcaniana que construyó un edificio con forma cónica que derramó coladas lávicas basálticas tipo "AA", así como flujos de escoria; y formó extensos campos de ceniza. Por su cuenta, el edificio volcánico del sureste del país también conocido como "Chichón", presentó una erupción explosiva de tipo freatopliniana, la cual pese a la evacuación previa, cobró más de dos millares de vidas y, cubrió de ceniza varias entidades federativas, evento que cuestionó el quehacer y las decisiones tomadas por el gobierno federal y estatal, los cuales fueron acusados por negligencia (Revista Proceso, 1982).

\section{Eventos de remoción en masa}

Por otra parte, coexisten procesos de remoción en masa o gravitacionales, a los cuales se integran los deslizamientos, hundimientos por desecación de suelos y abatimiento de mantos freáticos; subsidencias (relacionadas con hundimientos provocados por facturas y/o fallas geológicas, por la acumulación de sedimentos); flujos de suelos y/o rocas, reptación (creep); y caída libre de rocas y/o detritos. Este grupo tiene amplia representación en el territorio nacional debido a las condiciones topográficas predominantes. Este grupo tiene amplia representación en el territorio nacional debido a las condiciones topográficas predominantes pues, poco más de $1,400,000 \mathrm{~km}^{2}$ de éste corresponden a sistemas serranos compuestos por laderas de geometría cóncava, convexa y mixta, así como por rampas de piedemonte, abanicos aluviales y conos de deyección entre otras geoformas.

Aestas condiciones se le suman las características de los sustratos geológicos y la disposición que tienen estos a favor de la pendiente, a los cortes de origen antrópico y a la ocurrencia de ciclones, depresiones tropicales y frentes fríos que aportan suficiente agua para para la remover rocas y suelos (Sánchez et al., 2011; Galindo y Alcántara, 2015).

Entre los eventos gravitacionales más importantes registrados en la historia de México se cuentan con los constantes hundimientos de la ciudad de México reportados desde el año 1521 y que afectan de manera continua la infraestructura de la misma en el tiempo presente; la caída de bloques en el poblado de Santa María Tixmadejé, Estado de México como efecto encadenado del sismo de Acambay ocurrido en el año de 1912 que tuvo magnitud de 7.4 (Urbina y Camacho, 1913; Monroy y Novelo 2010). Se circunscribe a este grupo, el rompimiento de las presas de jales de la mina Dos Estrellas en Tlalpujahua Michoacán conocido como "Las lamas" ocurrido en 1937, el cual sepultó a una localidad conocida como Real de minas de Tlalpujahua (Macías et al., 2015; Corona et al., 2017).

De manera reciente se cuenta con el efecto de represamiento de río Los Venados en el estado de Hidalgo y los procesos de socavación lateral que a su vez provocaron deslizamientos sobre planos predispuestos en la localidad de Meztitlán Hidalgo que provocó el Huracán Arlene; así como deslizamientos múltiples en las sierras de Oaxaca y Chiapas (1995); en la Sierra Norte de Puebla (1997); en la margen derecha del río Grijalva (2007); en el sistema montañoso del poblado de Mesón Viejo en Temascaltepec, México (2010); en Motozintla, Chiapas (2012); en 
Pijijiapan, Chiapas (2012); en la carretera Tijuana-Ensenada (2013); en Tijuana Baja California (2018) y los acontecimientos en el poblado de San Gabriel en Jalisco (2019). Durante el año 2020 se registran eventos en el estado de Coahuila (carretera Puerto México - Ojo Caliente), en Fortín de las flores, en Xalapa, Coscomatepec, Huatusco y Coatepec, Veracruz; en Acapulco, Guerrero.

\section{Eventos hidrometeorológicos}

En otro orden de complejidad y dinámica, los riesgos hidrometeorológicos sobresalen por el número de ocurrencia, frecuencia y daños ocasionados en regiones costeras correspondientes al Mar Caribe, Golfo de México y costas del Pacífico las cuales son afectadas por huracanes y depresiones tropicales. En las regiones centro, meridional y septentrional cuando se registran precipitaciones extraordinarias e inundaciones, las cuales son favorecidas por frenes fríos y "nortes", así como por influencia antrópica que provoca cambios de configuración de la red fluvial, la saturación de ductos y la mala planeación geográfica urbana entre otras.

En este ámbito, existen evidencias presencia de heladas, sequías e inundaciones que datan de los años de 1543, 1557, 1599, 1620, 1621, 1691, 1785, 1786, 1808 y 1809 y que afectaron diversas regiones del país; así como una onda gélida reportada en 1818.

En el campo de los huracanes, el abanico de eventos es amplio, y en la historia nacional ocurrieron algunos que, debido a la categoría, trayectoria, precipitación generada, oleaje de tormenta y daños ocasionados quedaron inmortalizados.

Los registros sistemáticos de tormentas y ciclones tropicales datan aproximadamente de la década de los años treinta. A partir de esta época se destacan entre otros Huracán Janet que impactó en Chetumal Quintana Roo en 1955; el Huracán Beuhlau considerado el primero en su tipo con Categoría 5 que tuvo una trayectoria hacia la Península de Yucatán; el Huracán Gilberto que desarrolló la misma potencia que impactó a la Península de Yucatán, Nuevo León, Tamaulipas y sur sureste de Estados Unidos (1988). Se suman a la lista Roxana (1995) que tuvo una trayectoria modificada por un sistema de alta presión y lo desvió hacia el sur en el Golfo de México; el Huracán Paulina ocurrido en 1997 y que devastó la costa guerrerense de Acapulco con Categoría 4.

Por su parte, el Huracán Mitch (1998) fue considerado como uno de los más poderosos registrados hasta la fecha; afectó por una trayectoria irregular a Guatemala y el resto de Centroamérica; el Huracán Stan (2005) que perturbó a la Península de Yucatán, Chiapas y Oaxaca; y que obligado a modificar su trayectoria debido a un sistema de alta presión colisionó con Guatemala y Belice; y el Huracán Katrina que afectó también a la región de Louisiana en Estados Unidos (Rosengaus, 1988; Castro 2010; Rosengaus et. al., 2014).

En 2006 se desarrolló el huracán Wilma afectando la Costa de Quintana Roo y Yucatán provocando tan solo en la Isla de Cozumel, una precipitación de 2,000 milímetros diarios durante dos días continuos; así como Ingrid y la tormenta tropical Manuel gestados en septiembre de 2013 que impactaron de forma sincrónica las costas del Golfo de México y del Pacífico. El resto que conforma a los "más desastrosos" se observa clasificado por océano de origen, en la Tabla 3.

\begin{tabular}{ll|rl} 
& \multicolumn{2}{c}{ Atlántico } & \multicolumn{2}{c}{ Costa del Pacífico } \\
\hline Año & Nombre del meteoro & Año & Nombre del meteoro \\
\hline 1933 & Huracán de Tamaulipas & 1933 & Huracán de Guerrero \\
\hline 1955 & Gladys, Campeche & 1976 & Liza, Baja California Sur \\
\hline 1995 & Opal, Campeche & 1959 & México (Colima y Jalisco) \\
\hline 2002 & Isidoro, Península de Yucatán & 2002 & Kena; Puerto Vallarta, Jalisco \\
\hline 2005 & Emily, Nuevo León & 2005 & Dean, Quintana Roo \\
\hline 2013 & Ingrid, de Campeche a Tamaulipas & 2014 & Boris, de Chiapas a Jalisco \\
\hline 2014 & Tt Dolly, Tamaulipas y Veracruz & 2015 & Patricia, Golfo de Tehuantepec a Jalisco \\
\hline 2017 & Tt Earl (Puebla, Veracruz e Hidalgo) & 2017 & Tt Lidia, Baja California Sur \\
\hline 2018 & Katya, costa del Golfo & 2019 & Lorena, Baja California Sur \\
\hline 2020 & Delta, Península de Yucatán & & \\
\hline
\end{tabular}

Tabla 3. Registro histórico de algunos de los huracanes y tormentas tropicales ( $\mathrm{Tt}$ ) de energía alta o concentrada que han generado problemas de inundaciones, deslizamientos, flujos de lodo y víctimas en México Fuente: Autor, 2021, con base en Castro 2010; Rosengaus et. al., 2014; NOAA, 2019. 
En la Figura 2, se muestra la distribución de los meteoros que afecta al espacio geográfico mexicano. Cabe destacar que en ésta se incluye el tema relacionado con los tornados, los cuales hasta hace poco tiempo han comenzado a ser estudiados de forma sistemática.

De estos últimos eventos, se cuentan por lo menos 126 eventos ocurridos entre el año 2000 y 2012, encontrándose a las entidades estatales con más eventos al Estado de México (14), Veracruz (12), Tlaxcala y Chiapas (11 de manera respectiva), Tamaulipas y Chihuahua (9 cada uno); Nuevo León y Tabasco con 6 en cada entidad; y 5 en Coahuila, Puebla y Quintana Roo (Macías y Avendaño, 2014). En la Tabla 3 se muestran algunas notificaciones de algunos tornados considerados como los más importantes.

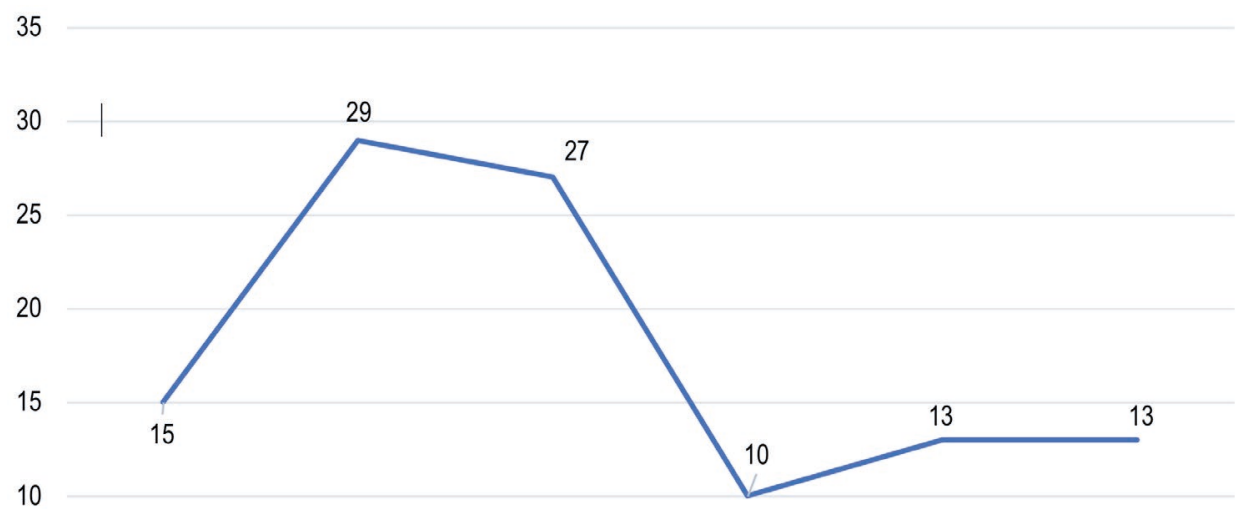

5

0
Granizo
Huracanes Inundaciones
Nevadas
Sequías
Tornados

Figura 2. Concentrado de eventos hidrometeorológicos más representativos ocurridos en México desde la época prehispánica hasta el tiempo presente

Fuente: Autor, 2021, con base en NOAA, 2019

En el gráfico se representan las inundaciones de la Gran Tenochtitlan -que involucra a la plaga de langostas- de 1446; así como las de 1454, 1629 y las correspondientes a 1856, 1951 y 2017 a la Ciudad de México; así como a la provocada por el desbordamiento del río Bravo en la frontera norte durante 1889, y las de 1949 ocurridas en Sonora y Sinaloa. En éste se incluyen dos de las regiones que poseen un mayor número de sucesos, la de Tlacotalpan en Veracruz (1774, 1932, 1937, 1944, 1969, 2010, 2017) y las de la ciudad de Villahermosa, Tabasco (1879, 1918 con 2 incidentes, 1927, 1932, 1944, 1952, 1955 y 2007); y se incorporan las del Valle de Chalco (2009 y 2010) y las más recientes ocurridas en la ciudad de Guadalajara, Jalisco.

Como eventos clasificados con el nombre de precipitación extraordinaria e inundaciones se cuenta con los reportes de 1653 y 1935 en el Distrito Federal, la de 1937 en Michoacán y las del año 2016 que provocaron también vientos a altas velocidades que afectaron las ciudades de Puebla, México, Toluca y Metepec en el Estado de México. Por su parte, las sequías han sido parte importante de la historia climática y meteorológica debido entre otras razones a procesos relacionados con retraso de temporada de lluvia, acortamiento del periodo de precipitación y presencia de canícula (Tabla 4).

Finalmente, en este ámbito, se registran granizadas y nevadas que han representado diferentes tipos de desastres en escala local. En el primer caso, la caída de granizo se asocia por lo general a sitios montañosos y de altitud superior a 2,000 metros, como es el caso de las Sierras Madre Occidental, Oriental, del Sur y Sistema Volcánico Transversal, aunque ello no excluye a cualquier otro sector territorial. Este tipo de precipitación se asocia con perforaciones y colapsos de techos en casas, comercios e industrias; condición inherente a víctimas humanas y, con la pérdida de cosechas entre otros efectos. Mientras tanto en el caso de las nevadas, éstas ocurren de manera usual en las cimas de los sistemas montañosos y en extensas áreas del norte del país; sin embargo, la presencia de éstas en otros territorios de menor altitud es posible y real, y representan problemas asociados con los sistemas de transporte, cortes de energía eléctrica, accidentes en vías carreteras, e hipotermias entre otros casos (Tabla 5). 


\begin{tabular}{|c|c|c|c|}
\hline \multicolumn{2}{|r|}{ Sequías } & \multicolumn{2}{|r|}{ Tornados } \\
\hline Año & Región & Año & Localización \\
\hline 1450 & La gran sequía de cuenca de México & 1521 & Tornado de Tlatelolco \\
\hline 1808 & $\begin{array}{l}\text { Sequía del Virreinato de la Nueva España } \\
(1808-1818)\end{array}$ & 2006 & Tornado de Tzintzuntzan Michoacán \\
\hline 1818 & $\begin{array}{l}\text { Sequía de Chiapas, Coahuila, Guerrero, } \\
\text { Nuevo León, Oaxaca, Aguascalientes, México } \\
\text { y Distrito Federal }\end{array}$ & 2007 & Tornado de Xalpatlahuaya, Tlaxcala \\
\hline 1860 & $\begin{array}{l}\text { Diversos estados del norte registran el evento } \\
\text { además de Veracruz, Chiapas y Oaxaca }\end{array}$ & 2007 & Tornado de Piedras Negras \\
\hline 1867 & $\begin{array}{l}\text { Sequía de San Luis Potosí, dura } \\
\text { aproximadamente tres años. }\end{array}$ & 2011 & Tornado de Jocotitlán, Estado de México \\
\hline 1870 & Sequía del noroeste y Yucatán & 2011 & Tornado en Atlacomulco, Estado de México \\
\hline 1877 & $\begin{array}{l}\text { Sequía de la mayor parte del territorio } \\
\text { nacional, centro y norte lo más afectado }\end{array}$ & 2012 & $\begin{array}{l}\text { Tornado de la Ciudad de México; "Tornado del } \\
\text { zócalo" }\end{array}$ \\
\hline 1884 & Sequía en todo el territorio nacional & 2014 & Tornado en San Cristóbal de las Casas \\
\hline 1892 & $\begin{array}{l}\text { Sequía de en todo el territorio (considerada la } \\
\text { peor del siglo; duración de 1892-1896) }\end{array}$ & 2015 & Tornado de Ciudad Acuña, Coahuila \\
\hline 1953 & $\begin{array}{l}\text { Considerada una de las más severas en el } \\
\text { territorio. }\end{array}$ & 2015 & $\begin{array}{l}\text { Tornado, Jiquipilco e Ixtlahuaca, Estado de } \\
\text { México }\end{array}$ \\
\hline 1977 & $\begin{array}{l}\text { Superó a la ocurrida } 24 \text { años atrás afectando } \\
\text { a todo el territorio nacional. }\end{array}$ & 2017 & Tornado de Huejotzingo, Puebla \\
\hline 1979 & $\begin{array}{l}\text { Sequía de Coahuila, duró } 10 \text { años; afectó a la } \\
\text { región del Altiplano }\end{array}$ & 2017 & Tornado de Riva Palacio, Chihuahua \\
\hline 2011 & $\begin{array}{l}\text { Afectó el centro y norte del país, considerada } \\
\text { como "extrema" }\end{array}$ & 2019 & Tornado de Morelia, Michoacán \\
\hline
\end{tabular}

Tabla 4. Histórico de riesgos hidrometeorológicos que comprenden a sequías (columna de la izquierda) y tornados (columna de la derecha)

Fuente: Autor, 2021, con base en Conagua, 2019.

\begin{tabular}{|c|c|c|c|}
\hline \multicolumn{2}{|r|}{ Granizadas } & \multicolumn{2}{|r|}{ Nevadas } \\
\hline Año & Región & Año & Localización \\
\hline 1976 & Distrito Federal & 1962 & Nuevo León, Monterrey \\
\hline 1994 & Aueyotlipan, Tlaxcala & 1967 & Norte y centro de México \\
\hline 1994 & Tula y Almoloya, Hidalgo & 1995 & Querétaro, Estado de México, Hidalgo \\
\hline 1996 & Calpulalpan, Estado de México & 1997 & Sonora, Chihuahua y Baja California Norte \\
\hline 1998 & Tijuana, Baja California & 1998 & Hidalgo, Tlaxcala \\
\hline 2000 & Zamora Michoacán & 2002 & Zacatecas \\
\hline 2001 & Fresnillo, Zacatecas y Guadalupe, Zacatecas & 2005 & Chihuahua \\
\hline 2001 & Allende y Villa Unión, Coahuila & 2006 & Baja California Norte (BCN), Chihuahua \\
\hline 2003 & Amecameca, Estado de México & 2007 & BCN, Chihuahua, Estado de México \\
\hline 2004 & Orizaba, Veracruz & 2008 & Baja California Norte \\
\hline 2005 & Altzayanca, Tlaxcala & & \\
\hline 2005 & Tulancingo y Omitlán, Hidalgo & & \\
\hline 2008 & Huimanguillo, Tabasco & & \\
\hline 2008 & Tlaquepaque, Jalisco & & \\
\hline 2019 & Guadalajara, Jalisco & & \\
\hline
\end{tabular}




\section{DISCUSIÓN}

La visión histórica de los geosistemas perturbadores ocurridos en el territorio nacional tuvo relativa significancia hasta que detonó durante el mes de septiembre de 1985 (Figura 3). Las experiencias locales y regionales demuestran que las condiciones que generan el riesgo y los desastres se relacionan con la integración de las variables que componen a la Ecuación General de Riesgo y que, la vulnerabilidad y la exposición representan los focos de atención prioritarios que requieren atención.

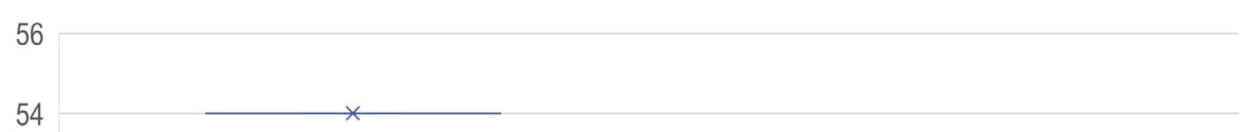

52

50

48

46

44

42

Figura 3. Gráfica que representa el porcentaje de los geosistemas perturbadores más frecuentes que han ocurrido en la República Mexicana

Fuente: Autor, 2021, con base en CENAPRED (2016, 2019), Bitácora personal (2019).

Después de muchos esfuerzos surgió la primera clasificación tipológica de los riesgos en México, creada y modificada por Cenapred (2010), codifica a los riesgos de tipo Astronómico (caída de meteoritos); Geológicos (que incluyen a los sismos, erupciones y emisiones volcánicas, tsunamis, inestabilidad de laderas, hundimientos regionales y locales, agrietamientos); a los Hidrometeorológicos (conformados por ciclones tropicales, inundaciones, tormentas de granizo, heladas y nevadas, viento, sequías, y frentes fríos); a los de tipo Químico-Tecnológicos (comprendidos por fugas y derrames, almacenamiento de sustancias peligrosas, incendios y explosiones, transporte de sustancias peligrosas); a los Sanitario-Ecológicos (formados por epidemias o plagas, erosión, contaminación de aire, agua, suelo y alimentos, residuos peligrosos); y a los Socio-Organizativos, que incluyen asociaciones-aglomeraciones masivas, grafiti*, sabotaje y terrorismo* (las marcadas con una notación, se incluyen o excluyen dependiendo de lineamientos administrativos); sin embargo, los trabajos relacionados con esta propuesta y todas aquellas de tipo legal y económico que se generaron a partir de ella, aún quedan cortas ante el tamaño de la necesidad de resolver de forma integral.

El problema global más importante se relaciona con el reconocimiento de que el $50 \%$ de la población se localiza en zonas de riesgo con la presencia-convivencia de una o más tipologías; y como el número de habitantes crece de manera alífera (Figura 4), la exposición y vulnerabilidad a los geosistemas perturbadores se incrementa de forma considerable; es decir, aproximadamente 60 y 65 millones de personas son proclives a sufrir la presencia de algún geosistema perturbador (Figura 4).

En este sentido, el Consejo Nacional de Población y Vivienda (CONAPO), estima que para el año 2040, la población alcanzará un número aproximado a los 130 millones de personas para descender de forma lenta y progresiva para la siguiente década;

A pesar de la condición establecida y los trabajos realizados por las instituciones nacionales, queda un sinsabor en el entendido de que todavía no es suficiente lo que se ha logrado hasta la fecha, por consiguiente, surgen preguntas que exigen respuestas como: ¿qué sigue?, ¿qué estamos dejando de observar?, ¿específicamente, en que se debe mejorar?, y otras más que envuelven la seguridad de los mexicanos. 


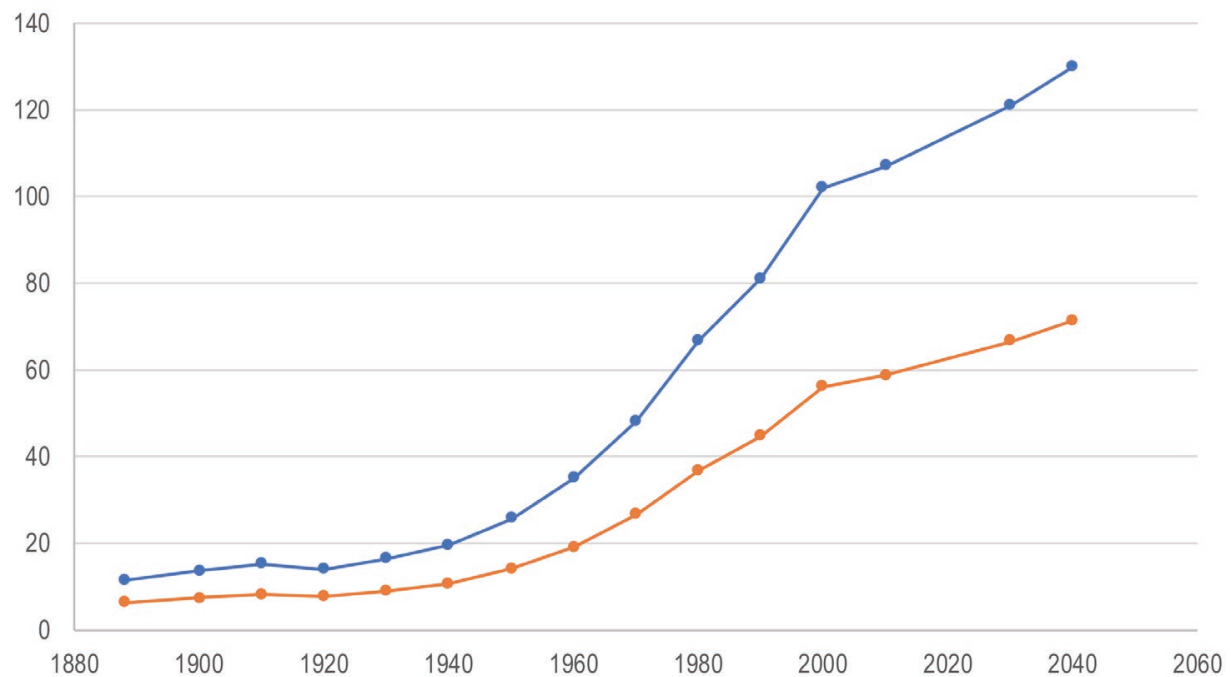

Figura 4. Gráfico de la población estimada de México registrada en los censos oficiales a partir del primer registro poblacional sistemático elaborado en 1885; y la proyección de Consejo Nacional de Población y Vivienda para 2040 (línea de arriba); y población estimada que habita en zonas de riesgo (línea de abajo)

Fuente: Autor, 2021, con base en datos de Rosenblat (1954 y 1967); Borah y Cook (1963); CEED (1970); CONAPO (2006); INEGI (2010), y World Populations Prospects (2019).

\section{CONCLUSIONES}

Como se ha establecido, la necesidad de reconocer el conjunto de desastres de origen natural que se han presentado en el territorio mexicano apunta a que la población y el gobierno puedan accionar de manera conjunta en un esquema real de gestión integral de los riesgos de desastre, más cuando se comprende que existe un aumento real del número de la población, la cual ocupará territorios susceptibles al desarrollo de geosistemas perturbadores, condición que aumentará la exposición y la vulnerabilidad de más de 65 millones de personas en 2040.

El conocimiento y concienciación social permitirá fortalecer el conjunto de acciones relacionadas con el aumento progresivo de las capacidades científicas, tecnológicas, sociales y gubernamentales de respuesta. Esto también permitirá contribuir al aumento de las condiciones de resiliencia en un escenario real al comprender características particulares de los geosistemas perturbadores, de los factores de enlace y el papel que desarrollan las condiciones del relieve y la hemerobia del medio ambiente.

Por otra, atacará problemas observados en el eslabón de los factores de tipo humano y territorial tales como los factores relacionados con la percepción y la educación; así como por la aceptación, adaptación comunicación del riesgo; legalidad, transparencia de los marcos jurídicos y el accionar de los responsables de la ejecución de planes y programas que favorecen la seguridad ciudadana.

En este orden de ideas, el proceso de concienciación de la información histórica, pretende fortalecer aspectos relacionados con el territorio y la distribución espacial de servicios y de la concentración de la población entre otros aspectos, desde una perspectiva geográfica integral que permite entre otras cosas, conocer condiciones de diagnosis, así como la observación y corrección de impactos territoriales; así como todas aquellas que se relacionan y obligan con la gobernanza.

Por otra parte, fortalece el esquema de la gestión integral de los riesgos y de los desastres, en la cual se reconocen los Objetivos del Desarrollo Sustentable (ODS), el Marco Sendai (MAS), el Acuerdo de París sobre el Cambio Climático, la Carta Iberoamericana en el Ámbito de la Gestión Pública y leyes nacionales como la General de Protección Civil, la Ley de Planeación, la Ley de Aguas Nacionales, la Ley de Desarrollo Rural Sustentable, la Ley General de Asentamientos Humanos, la Ley General de Cambio Climático y la Ley General de Responsabilidad Ambiental entre otros documentos de corte legal y de cooperación nacional e internacional.

Así, este trabajo puede ser un aporte para la planeación geográfica integral del territorio y en la planeación ejecución y seguimiento de propuestas de intervención local considerando aspectos relacionados con la reducción de los riesgos intensivos y extensivos que involucran a la gestión correctiva (riesgo existente), la gestión prospectiva (riesgo no existente en la actualidad); 
así como con la implementación de medidas físicas estructurales y no estructurales, y medidas socioeconómicas, ambientales e institucionales.

De manera particular, la concienciación de la información histórica apoya el desarrollo de la gestión compensatoria que involucra aspectos de preparación, respuesta, recuperación, transferencia y financiación del riesgo (riesgo aceptable); así como a la gestión local del riesgo que involucra acciones de participación y solidaridad ciudadana y atiende aspectos relacionados con los grupos de mayor vulnerabilidad social e integra contextos de orden identitario.

Estos grupos diferenciados de gestión permitirán construir políticas públicas serias que se enfoquen a resolver problemas reales del territorio y de la población mexicana y coadyuvarán a la seguridad de ésta y de sus pertenencias, las cuales se encontrarían por encima de intereses particulares o partidistas como ocurre en el tiempo presente.

\section{REFERENCIAS}

Alatorre-Ibargüengoitia, M. A., Delgado-Granados, H., \& Farraz-Montes, I. A. (2006). Hazard zoning for ballistic impact during volcanic explosions at Volcán de Fuego de Colima (México). In C. Siebe, J. L. Macías Gerardo, \& J. Aguirre-Díaz (Eds.), Neogene-Quaternary Continental Margin Volcanism: A perspective from México. Geological Society of America. https://doi.org/10.1130/2006.2402(o9)

Banco Mundial. (2018). Datos de libre acceso del Banco Mundial. Banco Mundial. Disponible en: http:// datos.bancomundial.org

Biondi, F., Galindo, I., Gavilanes, J., \& Elizalde, A. (2003). The growth response to the 1913 eruption of Volcán de Fuego de Colima. Quaternary Research, 59, 293-299.

Bitrán, D. (2001). Impacto socioeconómico de los principales desastres ocurridos en la República Mexicana en el año 20oo. Centro Nacional de Prevención de Desastres.

Castro, S. (2010). Variabilidad de los ciclones tropicales que afectan México. Interciencia, 35(4), 306-310.

Centro de Estudios Económicos y Demográficos CEED. (1970). Dinámica de la población de México. El Colegio de México.

Centro Nacional de Prevención de desastres CENAPRED. (2016). Impacto socio económico de los desastres en México. CENAPRED.

CENAPRED. (2017). Impacto socioeconómico de los principales desastres ocurridos en la República Mexicana durante 2016. CENAPRED.

Comisión Nacional del Agua CONAGUA. (2019). Monitor de sequía. CONAGUA

Confederación de Cámaras Nacionales de Comercio, Servicios y Turismo CONCANACO. (2016). Comunicados. Disponible en: www.concanaco.com.mx/category/comunicados

Consejo Nacional de Población y Vivienda. (2006). Proyecciones de la Población en México 2000-2050. En Virgilio Partida Bush (Ed). Secretaría de Gobernación.

Corona, P., Robles, J., Lozano, R., \& Martínez, M. (2017). Variaciones geoquímicas y mineralógicas en los jales del Distrito Minero Tlalpujahua- El Oro, México y sus implicaciones de impacto ambiental. Revista Mexicana de Ciencias Geológicas 34(3), 250-273.

Cruz, F. \& Rojas, C. (2009). El terremoto en México: estudio de caso. En Conferencia Internacional sobre Mitigación de Desastres en Instalaciones de Salud. Organización Panamericana de la Salud 2628. OPS.

Díaz, J. (2013). Los suelos volcánico-lacustres de la ciudad de México. Revista Internacional de Desastres Naturales, 6(2), 1-44.

Goff,F., Janik, C., \& Delgado, H. (1988). Geochemical surveillance of magmatic volátiles a Popocatépetl volcano, Mexico. Geological Society of America Bulletin, 110(6), 695-710.

Hernández, J., Ortiz, M., \& Zamorano, J. (1995). Regionalzación morfoestructural de la Sierra Madre del Sur, México. Investigaciones Geográficas, 31, 45-67.

Levi, L. \& Toscana, A. (2017). Vulnerabilidad en Tlatelolco a tres décadas de los sismos de 1985. Política y Cultura, 45, 125-152. http://ref.scielo.org/2cr3gg

Macías, J. \& Avendaño, A. (2014). Climatología de tornados en México. Investigaciones Geográficas, $83(1), 75-88$. 
Macías, J., Corona, P., Sánchez, J., Martínez, J., Garcí,a M., Cisneros, F. \& Garduño, V. (2015). The May 27, 1937 Catastrophic flow failure of gold tailings at Tlalpujahua, Michoacan Mexico. Natural Hazards and Earth System Sciences, 15(1), 1069-1085.

Monroy, S. \& Novelo D. (2010). Global vulnerability assessment in Santa Maria Tixmadeje, Estado de Mexico. American Geophysical Union, Fall Meeting 2010, 31(12), 1354.

Montemayor, C. (1991). Guerra en el paraíso. Diana.

National Geophysical Data Center, World Data Service NGDC/WDS. (2015). Global Historical Tsunami Database. National Geophysical Data Center.

National Oceanic and Atmospheric Administration NOAA. (2019). Tropical Cyclone Reports. National Hurricane Center, Central Pacific Hurricane Center.

Pérez, J., \& García, V. (1989). Y volvió a temblar. Cronología de los sismos en México. Cuadernos de la Casa Chata, Secretaría de Educación Pública, 135(1), 204.

Petróleos Mexicanos PEMEX. (1980). Informe de los trabajos realizados para el control del Pozo Ixtoc-1, el combate del derrame de petróleo y determinación de sus efectos sobre el ambiente marino. CENSA, INE, \& CIE.

Robin, C. Mossand, P., Camus, G. \& Cantagrel, J. (1981). Eruptive history of the Colima volcanic complex. Journal of Volcanology, 31(2), 99-113.

Robin, C. \& Boudal, C., (1989). A gigantic Bezymianny-typo evento at the beginning of modern volcán Popocatepetl. Journal of Volcanology and Geothermal Research, 31(2), 115-130.

Robin, C., Camus, G. \& Gourgaud, A. (1991). Eruptive and magmatic cycles at Fuego de Colima volcano (Mexico). Journal of Vulcanology and Geothermal Research, 45(3-4), 209-225.

Rosengaus, M. (1988). Efectos destructivos de ciclones tropicales. MAPFRERE.

Rosengaus, M., Jiménez, M. \& Vázquez, M. (2014). Atlas climatológico de ciclones tropicales en México. Instituto Mexicano de Tecnología del Agua IMTA \& CENAPRED.

Sánchez, J., Serrano, M., Sangermán, D. Navarro, A., Vera, G. \& Cuevas, J. (2011). Extreme hydrometeorological events and disasters in urban and rural communities in Motozintla, Chiapas. Revista Mexicana de Ciencias Agrícolas, 2, 167-181.

Siebe, C., Abrams, M. \& Macías, J. (1995). Derrumbes gigantes, depósitos de avalancha de escombros y edad del actual cono del volcán Popocatépetl. BV Salud.

Soto, L.A., Botello, A.V., Licea-Durán, S., Lizárraga-Partida, M.L. \& Yáñez-Arancibia, A. (2014). The environmental legacy of the Ixtoc-I oil spill in Campeche Sound, southwestern Gulf of Mexico. Front. Mar. Sci. 1, 57. http://doi.org/10.3389/fmars.2014.00057

Valdivia, L. \& Castillo, C. (2012). Los tsunamis en el Estado de Jalisco. Cuadernos de Geografía, 13(25), 1-55.

Verona, N. \& Ramírez, T. (2012). Técnicas histórico-etnográficas en la reconstrucción y caracterización de tsunamis: el ejemplo del gran tsunami del 22 de junio de 1932, en las costas del Pacífico mexicano. Revista Geografía Norte Grande, 53, 107-122. 